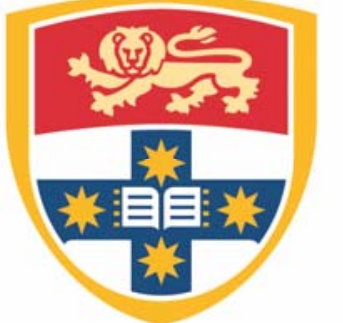

\title{
THE UNIVERSITY OF
}

SYDNEY

Economics Working Paper Series

$2015-3$

Soft Budget Constraints in Public Hospitals

Donald J. Wright

February 2015 


\title{
Soft Budget Constraints in Public Hospitals
}

\author{
Donald J. Wright*
}

February 2015

\begin{abstract}
A soft budget constraint arises when a government is unable to commit to not 'bailout' a public hospital if the public hospital exhausts its budget before the end of the budget period. It is shown that if the political costs of a 'bailout' are relatively small, then the public hospital exhausts the welfare maximising budget before the end of the budget period and a 'bailout' occurs. In anticipation, the government offers a budget to the public hospital which may be greater than or less than the welfare maximising budget. In either case, the public hospital treats "too many" elective patients before the 'bailout' and "too few" after. The introduction of a private hospital reduces the size of any 'bailout' and increases welfare.
\end{abstract}

Keyword: soft budget constraint, public hospital, welfare

JEL Codes: H4, I18

${ }^{*}$ School of Economics, Faculty of Arts and Social Sciences, University of Sydney, NSW, 2006, Australia, Ph: 61+2+93516609, Email: don.wright@sydney.edu.au. No conflicts of interest exist in the production of this work. I thank the two referees for their helpful comments. 


\section{Introduction}

A soft budget constraint exists if (i) an enterprise exhausts its budget before the end of the budget period and the funding source refinances the enterprise so that it can continue to operate for the remainder of the budget period and (ii) the enterprise knows that this refinancing will occur and makes choices based on this knowledge as does the funding source, Kornai (1986) and Maskin (1996). The essential feature of a soft budget constraint is the inability of the funding body to commit not to refinance the enterprise once the budget is exhausted, Dewatripont and Maskin, (1995).

Soft budget constraints are prevalent in the public hospital sector. Kornai (2009) argues that public hospitals often exhaust their budgets before the end of the budget period or run up debts by the end of the budget period knowing that the government will 'bail them out' by providing additional funding or paying off debts. Hagen and Kaarboe (2006) document that supplementary funding (bailing out) was common in the years preceding and following the Norwegian hospital reforms of 2002.

There is a small literature on soft budget constraints in public hospitals. Shen and Eggleston (2009) and Brekke, Siciliani, and Straume (2012) theoretically analyse the effect of soft budget constraints on the incentives for cost reductions and on quality provision in publicly funded hospitals. They find that soft budget constraints weaken the incentives public hospitals have for cost efficiency but have ambiguous effects on public hospital quality. Shen and Eggleston (2009) empirically examine these predictions using US data and find that softer budget constraints are associated with less cost efficiency and higher quality. Eggleston and Shen (2011) find that not-for profit and public hospitals are less cost efficient and have higher quality than for profit hospitals after controlling for the softness of budget 
constraints. All of these papers incorporate soft budgets into their analysis by assuming there is some probability that the government will bailout the public hospital if it exhausts its budget before the end of the budget period.

This paper differs from the existing literature by examining the conditions under which a 'bailout' occurs and by specifically incorporating the response of both the public hospital and the government to the existence of a soft budget constraint. The model has a simple structure. The government chooses the public hospital's budget and the public hospital chooses the number of non-elective and elective patients to treat. If the budget constraint is hard, the government gives the public hospital a budget which is just sufficient to fund the welfare maximising number of patients and the public hospital chooses to treat this number of patients.

On the other hand, if the budget constraint is soft, the government and the public hospital play a two period game over the budget period. In the first period, the government chooses a budget for the public hospital. The public hospital takes this budget as given and chooses the number of patients to treat and the proportion of the budget period in which to exhaust this budget. As a result, the length of the first period is chosen by the public hospital. If the public hospital chooses not to exhaust the budget before the end of the budget period, the game ends, otherwise a second period is entered. In the second period, the government chooses a budget for the public hospital, but now there are political costs of funding any 'bailout'. The public hospital then chooses the number of patients to treat.

Where the political costs of a 'bailout' are independent of the length of the bailout period, Proposition 1 states that if these political costs are relatively large, then the welfare maximising hard budget is not exhausted within the budget period, while if they are relatively small, this budget is 
exhausted within the budget period and a 'bailout' occurs.

Proposition 2 states that where the welfare maximising budget is exhausted within the budget period, the optimal period 1 budget may be greater or less than the welfare maximising budget. In either case, relative to the welfare maximum, there are 'too many' elective patients treated in period 1 and 'too few' elective patients treated in period 2.

The model is extended to allow the political costs of a 'bailout' to be an increasing function of the length of the 'bailout' period. In contrast to Proposition 1, it is shown that the welfare maximising hard budget is always exhausted before the end of the budget period and a 'bailout' occurs. This is stated in Proposition 3 while Proposition 4 states that the optimal period 1 budget may be greater or less than the welfare maximising budget. Once again, relative to the welfare maximum, there are 'too many' elective patients treated in period 1 and 'too few' treated in period 2 .

By optimally choosing the period 1 budget the government is able to somewhat offset the welfare reducing effect of the soft budget constraint. In the final section of the paper, the role that institutions play in affecting the size of any 'bailout' and welfare are explored. In particular, it is shown that the presence of a private hospital, that treats elective patients not treated by the public hospital, reduces the size of any 'bailout' and increases welfare.

\section{Basic Model - Hard Budget Constraint}

\section{$2.1 \quad$ Patients}

In the budget period, normalised to length 1, there are $E$ patients seeking elective treatment and $N$ patients seeking non-elective treatment at a public hospital. These patients arrive at a constant rate throughout the period. 
The benefit any non-elective patient receives from treatment is $b_{n}$ whereas the benefit elective patient $i$ receives from treatment is patient specific and given by $b_{i}$.

\subsection{The Public Hospital}

The cost to the public hospital of treating patients of any type is assumed constant and given by $c$. Elective patients are ordered from highest to lowest benefit from treatment. It is assumed that the total benefit from treating the $e$ patients with the highest benefits can be represented by a differentiable total benefit function $b(e)$, where $b^{\prime}(e)>0$ and $b^{\prime \prime}(e)<0$ by the ordering of patients. The public hospital is given a budget $B$ by the government and the hospital chooses the number of non-elective patients, $n$, and elective patients, $e$, to treat to maximise total patient benefit, $H$, subject to the cost of treating patients being no greater than $B$ and the number of nonelective and elective patients being no greater than $N$ and $E$, respectively. It is assumed that the public hospital knows the benefit a particular patient derives from treatment and so if constrained by its budget to treat less than $N+E$ patients, it treats those patients with the highest benefit. The public hospital's problem is

$$
\max _{n, e} H \equiv b_{n} \cdot n+b(e)
$$

subject to

$$
c \cdot n+c \cdot e \leq B
$$

and

$$
e \leq E, \quad n \leq N
$$

Let the solution to this problem be given by $n^{h}$ and $e^{h}$. 


\subsection{The Government}

The government chooses the public hospital's budget, $B$, to maximise total patient benefit. It is instructive to solve for the welfare maximising choice of $n$ and $e$. The welfare maximising problem is

$$
\max _{n, e} W \equiv b_{n} \cdot n+b(e)-(c \cdot n+c \cdot e)
$$

subject to

$$
n \leq N, \quad e \leq E
$$

Some additional assumptions are made to help solve this problem.

Assumption 1: $b_{n}-c>b^{\prime}(0)-c>0$. This assumption ensures that all non-elective and some elective patients are treated.

Assumption 2: $b^{\prime}(E)-c<0$. Together with Assumption 1, this ensures an interior solution for $e$, that is, $e<E$.

Let the solution to the welfare maximising problem be $n^{*}$ and $e^{*}$. This solution is given by

$$
n^{*}=N
$$

and

$$
b^{\prime}\left(e^{*}\right)-c=0 .
$$

This last equation is the familiar condition that welfare is maximised where marginal benefit equals marginal cost. The strict concavity of $b(e)$ ensures that this is a maximum.

Let $B^{*}=c \cdot n^{*}+c \cdot e^{*}$. If $B^{*}$ is given to the public hospital then it will choose $n^{h}=n^{*}=N$ because of Assumption 1. It will then spend $B^{*}-c \cdot N$ (the remaining budget) on treating elective patients and because $b(e)$ is an increasing function it will choose $e^{h}=e^{*}$. Therefore, under a hard budget constraint the government can achieve the welfare maximising solution by providing the public hospital a budget of $B^{*}$. 


\section{Soft Budget Constraint}

In this paper, as in Dewatripont and Maskin (1995), a soft budget constraint arises through a failure of commitment by the funding agency. In particular, if the public hospital's budget is exhausted before the end of the budget period, the government provides additional funding to the public hospital so it can continue to treat patients as they arrive. The public hospital understands the incentives of the government and the government understands the incentives of the public hospital and both behave accordingly.

The timing of the game is as follows. In period 1, the government chooses a budget, $B_{1}$, for the public hospital. Given $B_{1}$, the public hospital chooses the proportion of the budget period, $\phi \in[0,1]$, in which it exhausts this budget. Simultaneously with its choice of $\phi$, the public hospital chooses how many elective patients, $e_{1}$, and non-elective patients, $n_{1}$, to treat. If $\phi=1$, the game ends. If $\phi<1$, the game has a second period, which has length $(1-\phi)$. In period 2 , the government chooses a budget, $B_{2}$, for the public hospital and given $B_{2}$, the public hospital chooses how many elective patients, $e_{2}$, and non-elective patients, $n_{2}$, to treat. The game ends.

It is assumed that all patients arrive at a constant rate throughout periods 1 and 2. In addition, it is assumed that the composition of elective patient benefits in any period is the same as it is in the full budget period,

that is, the benefit of the $e_{1}^{t h}$ patient in period 1 is the same as the $e^{\text {th }}$ patient's in the full period, where $e_{1}=\phi e$. As a result, $b^{\prime}\left(\frac{e_{1}}{\phi}\right)=b^{\prime}(e)$, where $\frac{e_{1}}{\phi}=e$, and the total benefit of treating $e_{1}$ patients in period 1 is $b\left(\frac{e_{1}}{\phi}\right) \cdot \phi$.

\subsection{Period Two}

Given $\phi<1$, in period 2, the government provides the public hospital with a budget, $B_{2}$, in order for the hospital to keep treating patients as they 
arrive. It is assumed that the cost of funding $B_{2}$ is $(1+\gamma) \cdot B_{2}$, where $\gamma \geq 0$ reflects the political cost of transferring an additional unit of funding to the public hospital. Although not modeled, these political cost might represent the electoral damage of a 'bailout' which are increasing in the size of the 'bailout', that is, in the size of $B_{2}$. A similar assumption is made by Robinson and Torvik (2009), where the cost for a politician of transferring one more unit of resources to a particular group is $1+\gamma$.

It is instructive to solve for the government's optimal choice of the number of non-elective and elective patients to treat, $n_{2}$ and $e_{2}$, respectively. The government's problem is

$$
\max _{n_{2}, e_{2}} W_{2} \equiv b_{n} \cdot n_{2}+b\left(\frac{e_{2}}{1-\phi}\right) \cdot(1-\phi)-(1+\gamma) \cdot\left(c \cdot n_{2}+c \cdot e_{2}\right)
$$

subject to

$$
n_{2} \leq(1-\phi) \cdot N, \quad e_{2} \leq(1-\phi) \cdot E .^{1}
$$

Let the solution to the government's problem be $\hat{n}_{2}$ and $\hat{e}_{2}$. There are three cases to consider.

(i) If $(1+\gamma) \cdot c>b_{n}>b^{\prime}(0)$, then the marginal cost of treating patients exceeds the marginal benefit of treating both types of patients and the solution to the government's problem is $\hat{n}_{2}=0$ and $\hat{e}_{2}=0$.

(ii) If $b_{n} \geq(1+\gamma) \cdot c \geq b^{\prime}(0)$, then the marginal benefit of treating nonelective patients exceeds marginal cost and $\hat{n}_{2}=(1-\phi) \cdot N$ while $\hat{e}_{2}=0$.

(iii) If $b_{n}>b^{\prime}(0)>(1+\gamma) \cdot c$, then the marginal benefit of treating some elective patients exceeds marginal cost and $\hat{e}_{2}>0$ satisfies

$$
b^{\prime}\left(\frac{\hat{e}_{2}}{1-\phi}\right)-(1+\gamma) \cdot c=0
$$

\footnotetext{
${ }^{1}$ It turns out that elective patients not treated in period 1 have treatment benefit less than $c$. As a result, in period 2, the government only chooses to treat elective patients that arrive in period 2 .
} 
with $\hat{n}_{2}=(1-\phi) \cdot N$. In the Appendix, it is shown that $\frac{d \hat{e}_{2}}{d \gamma}<0$, that is, an increase in $\gamma$ decreases the number of elective patients treated in period 2. Let $\hat{e}$ solve $b^{\prime}(\hat{e})=(1+\gamma) \cdot c$, then $\hat{e}_{2}=(1-\phi) \cdot \hat{e}$. As expected, this solution involves the government choosing the number of non-elective and electives patients to be the fraction $(1-\phi)$ of what it would have chosen for the whole period, given $\gamma$.

The intuition is clear. Increases in $\gamma$ increase the marginal cost of treatment. As this marginal cost rises, less elective patients are treated until it is so large that no elective patients are treated. Further increases in marginal cost have no effect on the number of patients treated until it becomes so large that it is greater than the marginal benefit of treating non-elective patients. At this point, no patients are treated in period 2 .

Let $\hat{B}_{2}=c \cdot \hat{n}_{2}+c \cdot \hat{e}_{2}$. If the government gives the public hospital $\hat{B}_{2}$ in each case above, then the public hospital chooses to treat the number of patients that is optimal from the government's perspective for each case. For example, if marginal benefits, marginal cost, and $\gamma$ are as given in case (ii), then a budget of $\hat{B}_{2}=c \cdot(1-\phi) \cdot N$ induces the public hospital to treat $(1-\phi) \cdot N$ non-elective patients in period 2 .

Denote the public hospital's benefit from being given the welfare maximising budget in period 2 by $\hat{H}_{2}(\gamma)$. From above

$$
\hat{H}_{2}(\gamma)= \begin{cases}0 ; & \text { if case (i) } \\ b_{n} \cdot(1-\phi) \cdot N ; & \text { if case (ii) } \\ b(\hat{e}) \cdot(1-\phi)+b_{n} \cdot(1-\phi) \cdot N ; & \text { if case (iii) }\end{cases}
$$

\subsection{Period One}

In period 1 the government gives the public hospital a budget and then the public hospital chooses when to exhaust the budget and how many elective 
and non-elective patients to treat.

\section{The Public Hospital's Problem}

The public hospital is given a budget, $B_{1}$, and chooses $n_{1}^{h}, e_{1}^{h}$, and $\phi$ to maximise total patient benefit taking into account the effect its choice of $\phi$ has on its period 2 benefit. Its problem is,

$$
\max _{n_{1}^{h}, e_{1}^{h}, \phi} H_{1} \equiv b_{n} \cdot n_{1}^{h}+b\left(\frac{e_{1}^{h}}{\phi}\right) \cdot \phi+\hat{H}_{2}(\gamma)
$$

subject to

$$
c \cdot n_{1}^{h}+c \cdot e_{1}^{h} \leq B_{1}
$$

and

$$
n_{1}^{h} \leq \phi \cdot N, \quad e_{1}^{h} \leq \phi \cdot E .
$$

As long as $B_{1} \leq c \cdot N+c \cdot E$ constraint (13) binds as $H_{1}$ is increasing in $n_{1}^{h}$ and $e_{1}^{h}$. Given that (13) binds, $n_{1}^{h}=\phi \cdot N$ otherwise $n_{1}^{h}$ could be increased and $e_{1}^{h}$ decreased so that (13) continues to bind and yet $H_{1}$ would be greater. Therefore, all non-elective patients that arrive in period 1 are treated. Given these two binding constraints, $e_{1}^{h}=\frac{B_{1}-c \cdot \phi \cdot N}{c}$. Substituting these equalities into $H_{1}$ yields

$$
H_{1}\left(\phi, B_{1}\right)=b_{n} \cdot \phi \cdot N+b\left(\frac{B_{1}}{c \cdot \phi}-N\right) \cdot \phi+\hat{H}_{2}(\gamma)
$$

In the Appendix it is shown that $H_{1}(\cdot)$ is concave in $\phi$. Let the solution to the public hospital's choice of $\phi$ be denoted by $\hat{\phi}_{1}^{h}$. Proposition 1 below establishes the conditions under which the welfare maximising hard budget, $B^{*}$, is exhausted before the end of the budget period.

Proposition 1: (i) Let $(1+\gamma) \cdot c>b_{n}>b^{\prime}(0)$. If the public hospital is given the welfare maximising hard budget, $B^{*}$, it chooses $\hat{\phi}_{1}^{h}=1$ and does not exhaust the budget in the budget period. 
(ii) Let $b_{n} \geq(1+\gamma) \cdot c \geq b^{\prime}(0)$. (a) If the public hospital is given $B^{*}$ and $b\left(e^{*}\right)-b^{\prime}\left(e^{*}\right) \cdot\left(e^{*}+n^{*}\right) \geq 0$, then it chooses $\hat{\phi}_{1}^{h}=1$ and does not exhaust the budget in the budget period. (b) If $b\left(e^{*}\right)-b^{\prime}\left(e^{*}\right) \cdot\left(e^{*}+n^{*}\right)<0$, then the public hospital chooses $\hat{\phi}_{1}^{h}<1$ and exhausts $B^{*}$ before the end of the budget period.

(iii) Let $b_{n}>b^{\prime}(0)>(1+\gamma) \cdot c$. (a) If the public hospital is given $B^{*}$ and $b\left(e^{*}\right)-b^{\prime}\left(e^{*}\right) \cdot\left(e^{*}+n^{*}\right) \geq 0$, then there exists a critical $\gamma, \gamma_{c}$, such that for $\gamma \geq \gamma_{c}$, the public hospital chooses $\hat{\phi}_{1}^{h}=1$ and does not exhaust the budget in the budget period, while if $\gamma<\gamma_{c}$ it chooses $\hat{\phi}_{1}^{h}<1$ and does exhaust the budget before the end of the budget period. (b) If $b\left(e^{*}\right)-b^{\prime}\left(e^{*}\right) \cdot\left(e^{*}+n^{*}\right)<0$, then the public hospital chooses $\hat{\phi}_{1}^{h}<1$ and exhausts $B^{*}$ before the end of the budget period.

Proof: In the Appendix

The intuition is clear. For relatively large $\gamma$, case (i) above, the public hospital understands that the political costs of a 'bailout' are so large that no 'bailout' will occur. As a result, it chooses not to exhausts the budget so that it can treat all the non-elective patients that arrive.

For intermediate values of $\gamma$, case (ii) above, the public hospital understands that if it chooses to exhaust the budget a 'bailout' that finances the treatment of all non-elective patients will occur. Given $B^{*}$ and $\phi=1$, the trade-off the public hospital faces when decreasing $\phi$ is between treating less elective patients in period 2 (the period 2 quantity effect) and more elective patients in period 1 (the period 1 allocation effect). This trade-off is reflected in the condition $-b\left(e^{*}\right)+b^{\prime}\left(e^{*}\right) \cdot\left(e^{*}+n^{*}\right)$. For large $N=n^{*}$, reducing $\phi$ releases a large part of $B^{*}$ to treat additional elective patients in period 1. As a result, the allocation effect dominates and the budget is exhausted before the end of the budget period. 
For relatively small $\gamma$, case (iii) above, the public hospital understands that if it chooses to exhaust the budget a 'bailout' will occur. The period 2 quantity effect is now smaller as some elective patients are treated in period 2. The trade-off is now reflected in the condition $-\left(b\left(e^{*}\right)-b(\hat{e})\right)+b^{\prime}\left(e^{*}\right)$. $\left(e^{*}+n^{*}\right)$. For particularly small $\gamma$, the period 2 quantity effect is minimal ( $\hat{e}$ is close to $e^{*}$ ) and the budget is exhausted before the end of the budget period.

A Period One Comparative Static: The public hospital's choice of when to exhausts its budget is a function of the size of the budget and $\gamma$, that is, $\hat{\phi}_{1}^{h}\left(B_{1}, \gamma\right)$. Where the choice of $\phi$ is interior, it is shown in the Appendix

that $\frac{\partial \hat{\phi}_{1}^{h}}{\partial B_{1}}=\frac{\hat{\phi}_{1}^{h}}{B_{1}}>0$ and so $\frac{\partial \hat{\phi}_{1}^{h}}{\partial B_{1}} \cdot \frac{B_{1}}{\hat{\phi}_{1}^{h}}=1$. The elasticity of $\hat{\phi}_{1}^{h}$ with respect to $B_{1}$ equals 1 because of the proportionality of the elective patient marginal benefit function with respect to the length of period 1 .

Proposition 1 establishes conditions under which $B_{1}=B^{*}$ is exhausted by the public hospital before the end of the budget period, but what is the optimal period 1 budget from the point of view of the government?

\section{The Government's Problem}

The government's problem is to choose its period one budget to maximise the sum of patient benefits in periods 1 and 2 net of budget costs taking into account the public hospital's choice of $\hat{\phi}_{1}^{h}$ and its own choice of its period 2 budget.

The government's problem is

$$
\begin{aligned}
\max _{B_{1}} W_{1}\left(B_{1}\right) & =b_{n} \cdot \hat{\phi}_{1}^{h}\left(B_{1}\right) \cdot N+b\left(\frac{B_{1}}{c \cdot \hat{\phi}_{1}^{h}\left(B_{1}\right)}-N\right) \cdot \hat{\phi}_{1}^{h}\left(B_{1}\right) \\
& +\hat{H}_{2}\left(\gamma, \hat{\phi}_{1}^{h}\left(B_{1}\right)\right)-B_{1}-(1+\gamma) \cdot \hat{B}_{2}\left(\hat{\phi}_{1}^{h}\left(B_{1}\right)\right),
\end{aligned}
$$

where $b_{n} \cdot \hat{\phi}_{1}^{h}\left(B_{1}\right) \cdot N$ is the benefit from treating non-elective patients in period $1, b\left(\frac{B_{1}}{c \cdot \hat{\phi}_{1}^{h}\left(B_{1}\right)}-N\right) \cdot \hat{\phi}_{1}^{h}\left(B_{1}\right)$ is the benefit from treating $e_{1}^{h}$ elective 
patients in period $1, \hat{H}_{2}\left(\gamma, \hat{\phi}_{1}^{h}\left(B_{1}\right)\right)$ is optimal patient benefit in period 2 , and $\hat{B}_{2}=c \cdot\left(1-\hat{\phi}_{1}^{h}\left(B_{1}\right)\right) \cdot N+c \cdot\left(1-\hat{\phi}_{1}^{h}\left(B_{1}\right)\right) \cdot \hat{e}$. Let the solution to the government's problem be denoted $\hat{B}_{1}$. Proposition 2 below examines the relationship between $\hat{B}_{1}$ and $B^{*}$.

Proposition 2: (i) If the conditions of Proposition 1 for $B^{*}$ not to be exhausted before the end of the budget period are satisfied, then the optimal period 1 budget is $\hat{B}_{1}=B^{*}$ and the welfare maximum is achieved.

(ii) If the conditions of Proposition 1 for $B^{*}$ to be exhausted before the end of the budget period are satisfied, then the relationship between $\hat{B}_{1}$ and $B^{*}$ is ambiguous with $\hat{B}_{1}=0$ or $\hat{B}_{1}$ being chosen so that the public hospital finds it optimal to choose $\hat{\phi}_{1}^{h}=1$. In either case, the welfare maximum is not achieved.

Proof: In the Appendix.

Part (i) of Proposition 2 follows trivially from Proposition 1. If offered $B^{*}$, the public hospital chooses $\hat{\phi}_{1}^{h}=1$ and treats the welfare maximising number of non-elective and elective patients, $n^{*}=N$ and $e^{*}$, respectively. Therefore, the government sets $\hat{B}_{1}=B^{*}$ and achieves the welfare maximum.

In the Appendix it is shown that the relationship between $\hat{B}_{1}$ and $B^{*}$ is ambiguous because, at $B^{*}$, the sign of $\frac{d W_{1}}{d B_{1}}\left(B^{*}\right)$ is ambiguous. This ambiguity arises because of two opposing effects. If $B_{1}$ is increased it has the direct effect of increasing the number of elective patients treated in period 1. As "too many" elective patients are already treated in period 1 relative to the welfare maximum, $\hat{e}_{1}^{h}=\frac{B^{*}-c \cdot \hat{e}_{1}^{h} \cdot N}{c}=e^{*}+\left(1-\hat{\phi}_{1}^{h}\right) \cdot N>e^{*}>\phi \cdot e^{*}$, this effect decreases welfare. On the other hand, an increase in $B_{1}$ has the direct effect of decreasing the length of period 2 and decreasing $\hat{B}_{2}$. This directly reduces government costs and increases welfare. If the second effect dominates the first, then $\frac{d W_{1}}{d B_{1}}\left(B^{*}\right)>0$ and $\hat{B}_{1}>B^{*}$ (as long as $W_{1}\left(B_{1}\right)$ is concave). It 
should be noted that in this case $b_{n} \geq(1+\gamma) \cdot c$ so all non-elective patients that arrive in periods 1 and 2 are treated.

In the Appendix it is also shown that welfare, $W_{1}$, is a linear function of $B_{1}$. As a result, depending on the parameters of the model, the optimal period 1 budget is set so that it is immediately exhausted (where the cost of a 'bailout' is small), or it is set so that it is not exhausted in the budget period, (where the cost of a bailout is large). Finally, Numerical Example 1 is developed in the Appendix to show that either outcome is possible. ${ }^{2}$

\subsection{Endogenous Public Costs of Funds}

In the sections above, the government's cost of funds in period 2 was constant and equal to $\gamma$. This assumption led to the knife-edge result that $\hat{B}_{1}=0$ or $\hat{B}_{1}$ is such that the public hospital finds it optimal to choose $\hat{\phi}_{1}^{h}=1$. In this section, it is assumed that the government's costs of funds is a function of $\phi$, that is, $\gamma(\phi)$, where $\gamma^{\prime}(\phi)<0$ and $\gamma(1)=0$. The rationale for these restrictions is that the political cost of any 'bailout' is greater the longer is the 'bailout' period and as the length of this 'bailout' period approaches zero, the political costs of funds approaches 0 .

As $\phi$ is taken as given by the government in period 2, the analysis of period 2 is identical to that in section 3.1 above except $\gamma$ is replaced with $\gamma(\phi)$. Let the solution to the government's problem be given by $\check{e}_{2}$ and $\check{n}_{2}$ Define $\phi_{n}$ by $\left(1+\gamma\left(\phi_{n}\right)\right) \cdot c \equiv b_{n}$ and $\phi_{e}$ by $\left(1+\gamma\left(\phi_{e}\right)\right) \cdot c \equiv b^{\prime}(0)$. As in section 3.1 there are three cases to consider but now they are defined by the size of $\phi$.

(i) If $\phi<\phi_{n}$, then $\check{n}_{2}=0$ and $\check{e}_{2}=0$.

\footnotetext{
${ }^{2}$ The Mathematica notebooks for all the examples in this paper are available from the author on request.
} 
(ii) If $\phi_{n} \leq \phi \leq \phi_{e}$, then $\check{n}_{2}=(1-\phi) \cdot N$ and $\check{e}_{2}=0$.

(iii) If $\phi_{e}<\phi$, then $\check{n}_{2}=(1-\phi) \cdot N$ and $\check{e}_{2}>0$, where $\check{e}_{2}$ is the solution to

$$
b^{\prime}\left(\frac{\check{e}_{2}}{1-\phi}\right)-(1+\gamma(\phi)) \cdot c=0 .
$$

Let $\check{e}$ solve $b^{\prime}(\check{e})-(1+\gamma(\phi)) \cdot c=0$, then $\check{e}_{2}=(1-\phi) \cdot \check{e}$.

The government's optimal period 2 budget is $\check{B}_{2}(\phi)=c \cdot \check{n}_{2}+c \cdot \check{e}_{2}(\phi)$ and the public hospital's benefit $\check{H}_{2}(\phi)$ is

$$
\check{H}_{2}(\phi)= \begin{cases}0 ; & \text { if } \phi<\phi_{n} \\ b_{n} \cdot(1-\phi) \cdot N ; & \text { if } \phi_{n} \leq \phi \leq \phi_{e} \\ b(\check{e}(\phi)) \cdot(1-\phi)+b_{n} \cdot(1-\phi) \cdot N ; & \text { if } \phi_{e}<\phi\end{cases}
$$

The public hospital's problem in period 1 is

$$
\max _{\phi} \check{H}_{1}\left(\phi, B_{1}\right)=b_{n} \cdot \phi \cdot N+b\left(\frac{B_{1}}{c \cdot \phi}-N\right) \cdot \phi+\check{H}_{2}(\phi) .
$$

It is assumed that $b(\cdot)$ is sufficiently concave that $\check{H}_{1}(\cdot)$ is concave in $\phi$ (see the Appendix). Let the solution to the public hospital's choice of $\phi$ be denoted by $\check{\phi}_{1}^{h}$. Once again, as $b_{n}>b^{\prime}(0)>c$, all non-elective patients that arrive in period 1 are treated.

Proposition 3: If the public hospital is given $B^{*}$, then

(i) $\check{\phi}_{1}^{h}<1$, that is, $B^{*}$ is exhausted before the end of the budget period,

(ii) $\phi_{n} \leq \check{\phi}_{1}^{h}$, that is, all non-elective patients are treated, and

(iii) either $\check{\phi}_{1}^{h} \leq \phi_{e}$ in which case only non-elective patients are treated or $\phi_{e}<\check{\phi}_{1}^{h}$ in which case some elective patients are treated as well.

Proof: In the Appendix.

The first part of Proposition 3 establishes that if the government gives the public hospital the welfare maximising hard budget, $B^{*}$, then the public 
hospital exhausts this budget before the end of the budget period. This contrasts with Proposition 1 where conditions are given under which this budget is not exhausted in the budget period. The crucial difference is that in Proposition $3 \gamma(1)=0$ and there is no cost to the public hospital of reducing $\phi$ below $\phi=1$. The second and third parts of Proposition 3 establish that, given $B^{*}$, the public hospital's choice of $\phi$ always results in all non-elective patients being treated and might result in some elective patients being treated as well.

Period 1 government welfare is identical to (16) except for $\gamma\left(\check{\phi}_{1}^{h}\left(B_{1}\right)\right)$, that is,

$$
\begin{aligned}
\max _{B_{1}} \check{W}_{1}\left(B_{1}\right) & =b_{n} \cdot \check{\phi}_{1}^{h}\left(B_{1}\right) \cdot N+b\left(\frac{B_{1}}{c \cdot \check{\phi}_{1}^{h}\left(B_{1}\right)}-N\right) \cdot \check{\phi}_{1}^{h}\left(B_{1}\right) \\
& +\check{H}_{2}\left(\gamma\left(\check{\phi}_{1}^{h}\left(B_{1}\right)\right), \check{\phi}_{1}^{h}\left(B_{1}\right)\right) \\
& -B_{1}-\left(1+\gamma\left(\check{\phi}_{1}^{h}\left(B_{1}\right)\right)\right) \cdot \check{B}_{2}\left(\check{\phi}_{1}^{h}\left(B_{1}\right)\right) .
\end{aligned}
$$

Let the solution to the government's problem be denoted $\check{B}^{1}$.

Proposition 4: If the cost of public funds is endogenous, then (i) $\check{B}_{1} \geq$ $c \cdot \phi_{n} \cdot N$ and (ii) the relationship between $\check{B}_{1}$ and $B^{*}$ is ambiguous and the welfare maximum is not achieved.

Proof: In the Appendix.

An implication of Proposition 4 (i) is that in equilibrium the public hospital chooses $\phi \geq \phi_{n}$. Therefore, all non-elective patients that arrive in period 1 are treated and in addition all non-elective patients that arrive in period 2 are treated because $b_{n} \geq\left(1+\gamma\left(\phi_{n}\right)\right) \cdot c$.

In section 9 of the Appendix, it is shown that there are additional benefits and costs to increasing $B_{1}$ compared to subsection 3.2. An increase in $B_{1}$ increases $\phi$ and so reduces the public cost of funds $\gamma$. This directly increases 
welfare as any 'bailout' is less costly, but indirectly increases the size of the 'bailout' and decreases welfare to the extent that the number of electives patients treated in period 2 increases. As a result, the sign of $\frac{d \check{W}_{1}}{d B_{1}}\left(B^{*}\right)$ is ambiguous as is the relationship between $\check{B}_{1}$ and $B^{*}$.

It is also shown in the Appendix that, unlike in section 3.2, welfare is not a linear function of $B_{1}$. Nevertheless, in the region $\phi_{n} \leq \phi \leq \phi_{e}$, welfare can be an increasing function of $B_{1}$ as the indirect effect mentioned above is absent. Therefore, the case that is emphasized is the interesting case where both non-elective and elective patients are treated in period 2. This arises when $B_{1}$ is such that $\phi$ is chosen to be in the region $\phi_{e} \leq \phi$. In this case, Numerical Example 2 is developed in the Appendix to show that $\check{B}_{1}$ can be greater than or less than $B^{*}$. In particular, under the assumption that $\gamma(\phi)$ takes the form $\gamma=\gamma_{0}-\gamma_{0} \cdot \phi$, it is shown that $\check{B}_{1}>B^{*}$ if $\gamma_{0}$ is relatively small. For relatively small $\gamma_{0}, \check{e}_{2}$ is relatively large. As a result, the direct effects that increasing the period one budget have on the cost of the 'bailout' through reducing the length of the 'bailout' period (increasing $\phi$ ) and reducing the political cost of the 'bailout' (decreasing $\gamma$ ) more than offset the indirect effects that operate through increasing the number of elective patients treated in period 2 .

Regardless of whether $\check{B}_{1}$ is greater than or less than $B^{*}$, as long as $\check{\phi}_{1}^{h}<1$ "too many" elective patients are treated in period 1 and "too few" are treated in period 2 relative to the welfare maximum.

\section{A Public and a Private Hospital}

It was seen above, in the case where the cost of government funds was endogenous, that the government anticipates its lack of commitment in period 2 and, even though it optimally chooses its period 1 budget, it is not able 
to achieve the welfare maximum. In this section, a private hospital is introduced and its effect on the size of any 'bailout' and welfare is examined. Intuitively, the presence of a private hospital, that treats patients who are left untreated by the public hospital, weakens the incentives the government has to 'bailout' the public hospital in period 2 and so reduces the size of any 'bailout' and increases welfare. This intuition is now examined.

\subsection{The Private Hospital}

A monopoly private hospital treats elective patients who are left untreated by the public hospital. Assume the marginal cost of treatment by the private hospital is constant and equal to $c$. Given the number of patients treated by the public hospital in period $2, e_{2}$, and the length of period $2,(1-\phi)$, the residual demand curve of the private hospital is given by $p\left(e_{2}^{m}\right)=b^{\prime}\left(\frac{e_{2}+e_{2}^{m}}{1-\phi}\right)$. The private hospital's profit maximisation problem is

$$
\max _{e_{2}^{m}} \Pi_{m}=b^{\prime}\left(\frac{e_{2}+e_{2}^{m}}{1-\phi}\right) \cdot e_{2}^{m}-c \cdot e_{2}^{m}
$$

with associated first order condition

$$
\frac{\partial \Pi_{m}}{\partial e_{2}^{m}}=b_{e}^{\prime \prime}\left(\frac{e_{2}+e_{2}^{m}}{1-\phi}\right) \cdot \frac{e_{2}^{m}}{1-\phi}+b^{\prime}\left(\frac{e_{2}+e_{2}^{m}}{1-\phi}\right)-c=0 .
$$

Let the solution to this first order condition be $e_{2}^{m}\left(e_{2}\right)$, where $e_{2}^{m^{\prime}}(\cdot)<0$. That is, the more elective patients that are treated by the public hospital in period 2 the less elective patients are treated by the profit maximising private hospital. The patient pays a price of $p\left(e_{2}^{m}\left(e_{2}\right)\right)$ to the private hospital for treatment.

\subsection{Period Two}

As the private hospital treats elective patients not treated by the public hospital, it is assumed that the parameters of the model and $\gamma(\phi)$ are such 
that the equilibrium involves the public hospital treating some, but not all, elective patients in period 2. Given $\phi$, the government's period two problem is given by

$\max _{n_{2}, e_{2}} \tilde{W}_{2} \equiv b_{n} \cdot n_{2}+b\left(\frac{e_{2}+e_{2}^{m}\left(e_{2}\right)}{1-\phi}\right) \cdot(1-\phi)-(1+\gamma(\phi)) \cdot\left(c \cdot n_{2}+c \cdot e_{2}\right)-c \cdot e_{2}^{m}\left(e_{2}\right)$

subject to

$$
n_{2} \leq(1-\phi) \cdot N, \quad e_{2}+e_{2}^{m}\left(e_{2}\right) \leq(1-\phi) \cdot E
$$

This problem is similar to that in section 3.3 except that the costs of treating $e_{2}^{m}$ patients in the private hospital must be accounted for and $b\left(\frac{e_{2}}{1-\phi}\right)$ is replaced with $b\left(\frac{e_{2}+e_{m 2}\left(e_{2}\right)}{1-\phi}\right)$ because the public and private hospitals now both treat patients. Differentiating (23) with respect to $e_{2}$ yields

$$
\begin{aligned}
\frac{\partial \tilde{W}_{2}}{\partial e_{2}} & =b^{\prime}\left(\frac{e_{2}+e_{2}^{m}\left(e_{2}\right)}{1-\phi}\right)-(1+\gamma(\phi)) \cdot c+\left(b^{\prime}\left(\frac{e_{2}+e_{2}^{m}\left(e_{2}\right)}{1-\phi}\right)-c\right) \cdot \frac{d e_{2}^{m}}{d e_{2}} \\
& =\left(b^{\prime}\left(\frac{e_{2}+e_{2}^{m}\left(e_{2}\right)}{1-\phi}\right)-c\right) \cdot\left(1+\frac{d e_{2}^{m}}{d e_{2}}\right)-\gamma(\phi) \cdot c
\end{aligned}
$$

It is assumed that $b(\cdot)$ is sufficiently concave that $\tilde{W}_{2}(\cdot)$ is also concave. Setting (25) equal to zero and solving yields the solution to the government's problem for the number of elective patients to treat. Denote this solution by $\tilde{e}_{2}$. The trade-off is clear, from the government's perspective, increasing the number of patients treated in the public hospital increases welfare because it reduces the monopoly distortion (too few patients treated - the first term in the second row of (25)), but on the other hand it decreases welfare as treating patients in the public hospital is more costly than it is in the private hospital (the second term in the second row of (25)).

Let $\tilde{e}$ solve $b^{\prime}\left(\tilde{e}+e^{m}(\tilde{e})\right)-(1+\gamma(\phi)) \cdot c+\left(b^{\prime}\left(\tilde{e}+e^{m}(\tilde{e})\right)-c\right) \cdot \frac{d e^{m}}{d e}=0$, where $e^{m}(e)$ solves the monopolist's first order condition $b_{e}^{\prime \prime}\left(e+e^{m}\right) \cdot e^{m}+$ $b^{\prime}\left(e+e^{m}\right)-c=0$. As above, $\tilde{e}_{2}=(1-\phi) \cdot \tilde{e}$ and $\tilde{e}_{2}^{m}=(1-\phi) \cdot e^{m}(\tilde{e})$. 
Proposition 5: The welfare maximising number of elective patients treated in the public hospital in period 2 is less when a private hospital is in the market than when it is not. That is, $\tilde{e}_{2}<\check{e}_{2}$. In addition, $\tilde{e}_{2}+e_{2}^{m}\left(\tilde{e}_{2}\right)<\check{e}_{2}$. Proof: In the Appendix.

At $\check{e}_{2}$, the monopoly distortion is so small that the cost saving from treating patients in the private hospital rather than the public hospital dominates the trade-off outlined above and results in the government choosing $\tilde{e}_{2}<\check{e}_{2}$.

If the government gives the public hospital a period 2 budget of $\tilde{B}_{2}=$ $c \cdot \hat{n}_{2}+c \cdot \tilde{e}_{2}$, then the public hospital chooses $\tilde{n}_{2}^{h}=\hat{n}_{2}$ and $\tilde{e}_{2}^{h}=\tilde{e}_{2}$. Note that $\tilde{B}_{2}<\check{B}_{2}$, that is, given $\phi$, the presence of the private hospital reduces the optimal period 2 budget of the government below what it would have been in the absence of the private hospital and so reduces the size of any 'bailout'.

\subsection{Period One}

Given $B_{1}$, the public hospital's problem for the choice of $\phi$ is identical to (19) in section 3.3 except $b(\check{e}(\phi)) \cdot(1-\phi)$ is replaced with $b(\tilde{e}(\phi)) \cdot(1-\phi)$ as the public hospital only values the patients it treats. The derivative of this problem is

$$
\begin{aligned}
\frac{\partial \tilde{H}_{1}}{\partial \phi} & =b\left(\frac{B_{1}}{c \cdot \phi}-N\right)-b^{\prime}\left(\frac{B_{1}}{c \cdot \phi}-N\right) \cdot \frac{B_{1}}{c \cdot \phi} \\
& -b(\tilde{e}(\phi))+(1-\phi) \cdot b^{\prime}(\tilde{e}(\phi)) \cdot \frac{d \tilde{e}}{d \phi} .
\end{aligned}
$$

It is assumed that $\tilde{H}_{1}(\cdot)$ is concave in $\phi$. Let the solution to the public hospital's problem be denoted by $\tilde{\phi}_{1}^{h}$.

Proposition 6 If $b^{\prime \prime \prime}(e)=0$, then $\tilde{\phi}_{1}^{h} \geq \check{\phi}_{1}^{h}$. That is, the presence of a private hospital in the market induces the public hospital to exhaust its 
budget later in the budget period than if there was no private hospital in the market.

Proof: In the Appendix.

The intuition seems clear. In the presence of a private hospital, the public hospital treats less elective patients than otherwise in period $2, \tilde{e}_{2}<\check{e}_{2}$. Therefore, the direct cost to the public hospital of increasing $\phi$ in terms of patient benefit lost is reduced, $b(\tilde{e})<b(\check{e})$ (the first term in the second row of (26) above). On the other hand, there is an indirect benefit of increasing $\phi$ as it reduces the public cost of funds, $\gamma(\phi)$, and increases the number of patients treated by the public hospital in period 2 (the second term in the second row of (26) above). As long as $\tilde{e}$ is more responsive to changes in $\phi$ than $\check{e}$, then the indirect benefit of an increase in $\phi$ is greater in the presence of a private hospital than in its absence. In the Appendix it is shown that this is the case if $b^{\prime \prime \prime}(e)=0$. As the presence of a private hospital reduces the cost of increasing $\phi$ and increases the indirect benefit of increasing $\phi$ it follows that $\tilde{\phi}_{1}^{h} \geq \check{\phi}_{1}^{h}{ }^{3}$. This shorter 'bailout' period reinforces the effect the presence of a private hospital has on reducing the size of any 'bailout'.

Setting (26) to zero and solving yields $\tilde{\phi}_{1}^{h}\left(B_{1}\right)$. On substitution, the government's period 1 problem is

$$
\begin{aligned}
\max _{B_{1}} \tilde{W}_{1} & =b_{n} \cdot N+b\left(\frac{B_{1}}{c \cdot \tilde{\phi}_{1}^{h}\left(B_{1}\right)}-N\right) \cdot \tilde{\phi}_{1}^{h}\left(B_{1}\right) \\
& +\left[b\left(\tilde{e}\left(\tilde{\phi}_{1}^{h}\left(B_{1}\right)\right)+e^{m}(\tilde{e})\right)-c \cdot e^{m}(\tilde{e})\right] \cdot\left(1-\tilde{\phi}_{1}^{h}\left(B_{1}\right)\right) \\
& -B_{1}-\left(1+\gamma\left(\tilde{\phi}_{1}^{h}\left(B_{1}\right)\right)\right) \cdot \tilde{B}_{2}\left(\tilde{\phi}_{1}^{h}\left(B_{1}\right)\right)
\end{aligned}
$$

This is a complicated problem with many effects to consider. Let the solution to this problem be denoted $\tilde{B}_{1}$. In the Appendix it is argued that

\footnotetext{
${ }^{3}$ The condition given in Proposition 6 is a sufficient condition. Linearity of the marginal benefit function is not necessary. This is clear from the proof in the Appendix
} 
the sign of $\frac{d \tilde{W}_{1}}{d B_{1}}\left(\check{B}_{1}\right)$ is ambiguous. Therefore the author is able to say little about the relationship between $\check{B}_{1}$ and $\tilde{B}_{1}$ in general. To proceed, Numerical Example 3 is developed in the Appendix, where it is assumed that $\gamma(\phi)$ takes the form $\gamma=\gamma_{0}-\gamma_{0} \cdot \phi$.

In this example, regardless of $\gamma_{0}, \tilde{B}_{1}<\check{B}_{1}$. Given $\tilde{\phi}_{1}^{h}$ is an increasing function of $B_{1}$, the fact that $\tilde{\phi}_{1}^{h}>\check{\phi}_{1}^{h}$ is a reflection of Proposition 6, namely, that the presence of a private hospital delays exhaustion of the period 1 budget by the public hospital. Together, the lower period 1 budget and the greater $\phi$ that arise through the presence of the private hospital reduce the period 1 distortion in the number of elective patients treated, $\tilde{e}_{1}<\check{e}_{1}$. In fact, $\tilde{e}_{1}$ is quite close to its welfare maximising quantity, $\tilde{\phi}_{1}^{h} \cdot e^{*}$. This reduction in the period 1 distortion is the main source of the welfare gain that results from the presence of the private hospital.

Although the presence of the private hospital reduces the distortion caused by treating "too many" patients in period 1 , it exacerbates the distortion caused by treating "too few" elective patients in period 2, $\tilde{e}_{2}+\tilde{e}_{2}^{m}<\check{e}_{2}$. However, as the reduction in the number of elective patients the public hospital treats in period 2 reduces the size of the 'bailout' it is this which increases $\phi$ and increases welfare overall.

Finally, it should be noted that when $\gamma_{0}$ is relatively large, $\gamma_{0}=10$, the number of elective patients treated by the public hospital in period 2 is close to zero. Essentially, the cost of public funds is so large that the government prefers elective patients be treated by the private hospital in period 2 .

\section{Conclusion}

This paper has shown that in the presence of a soft budget constraint, the public hospital chooses to exhaust the welfare maximising hard budget be- 
fore the end of the budget period if the government cost of funds is (i) constant and relatively low, or (ii) endogenous and increasing in the length of the 'bailout' period. In anticipation, the government offers the public hospital a budget which maybe greater than or less than the welfare maximising budget. In either case, the presence of a soft budget constraint reduces welfare, and relative to the welfare maximum results in "too many" elective patients being treated before the 'bailout' and "too few" being treated after the 'bailout'.

As emphasised by Dewatripont and Maskin (1995) soft budget constraints arise from a lack of commitment by the funding agency. In the context of public hospital funding, whether these hospitals are funded by block or global budgets, as assumed in this paper, or by some variant of activity based funding, the problem of commitment arises once a budget is exhausted or deficits get to large. This suggests policy makers should look elsewhere than at hospital reimbursement schemes when trying to harden public hospital budget constraints. In particular, this paper has shown that the introduction of a private hospital reduces the size of any 'bailout' and increases welfare. Another avenue for reducing the size of any 'bailout' is through the incentives provided to public hospital managers by their payment schemes. This is a rich area for future research. 


\section{References}

Brekke, K., Siciliani, L., and O. R. Straume, (2012), "Hospital Competition with Soft Budgets," Norwegian School of Economics Discussion Paper, SAM 6 2012, March.

Dewatripont, M., and E. Maskin, (1995), "Credit and Efficiency in Centralized and Decentralized Economies," Review of Economic Studies, 62, October, 541-555.

Eggleston, K., and Y. C. Shen, (2011), "Soft Budget Constraints and Ownership: Empirical Evidence from US Hospitals," Economics Letters, 110, 7-11.

Hagen, T. P., and O. M. Kaarboe, (2006), "The Norwegian Hospital Reform of 2002: Central Government Takes Over Ownership of Public Hospitals," Health Policy, 76, 320-333.

Kornai, J., (1986), "The Soft Budget Constraint," Kyklos, 39, 3-30.

Kornai, J., (2009), "The Soft Budget Constraint Syndrome in the Hospital Sector," Society and Economy, 31, 5-31.

Maskin, E., (1996), "Theories of the Soft Budget-Constraint," Japan and the World Economy, 8, 125-133.

Robinson, J. A., and R. Torvik, (2009), "A Political Economy Theory of the Soft Budget Constraint," European Economic Review, 53, 786-798.

Shen, Y. C., and K. Eggleston, (2009), "The Effect of Soft Budget Constraints on Access and Quality in Hospital Care," International Journal of Health Care Finance and Economics, 9, 211-232. 


\section{Appendix}

1. A Period Two Comparative Static: Applying the Implicit Function Theorem to (10) in the text yields

$$
\frac{d \hat{e}_{2}}{d \gamma}=(1-\phi) \cdot \frac{c}{b^{\prime \prime}(\cdot)}=(1-\phi) \cdot \frac{d \hat{e}}{d \gamma}<0
$$

by the concavity of $b(e)$.

2. Concavity of $H_{1}(\phi)$ : Differentiating (A-5) or (A-8) below with respect to $\phi$ yields

$$
\frac{\partial^{2} H_{1}}{\partial \phi^{2}}=b_{e}^{\prime \prime}(\cdot) \cdot \frac{B_{1}^{2}}{c^{2} \phi^{3}}<0
$$

by the concavity of $b(\cdot)$.

3. Proof of Proposition 1: (i) Let $(1+\gamma) \cdot c>b_{n}>b^{\prime}(0)$. In this case,

$$
H_{1}\left(\phi, B_{1}\right)=b_{n} \cdot \phi \cdot N+b\left(\frac{B_{1}}{c \cdot \phi}-N\right) \cdot \phi
$$

as $\hat{H}_{2}(\gamma)=0$. If $B_{1} \leq c \cdot N$, then the public hospital spends all its budget on non-electives patients as $b_{n}>b^{\prime}(0)$ and $\hat{\phi}_{1}^{h}=\frac{B_{1}}{c \cdot N}$. If $B_{1}>c \cdot N$ then as well as treating all non-electives patients it treats some elective patients as well. To treat all the non-elective patients the public hospital chooses $\hat{\phi}_{1}^{h}=1$. Now $B^{*}=c \cdot N+c \cdot e^{*}>c \cdot N$, so if the public hospital is given $B^{*}$, the public hospital chooses $\hat{\phi}_{1}^{h}=1$.

(ii) Let $b_{n} \geq(1+\gamma) \cdot c \geq b^{\prime}(0)$. In this case,

$$
H_{1}\left(\phi, B_{1}\right)=b_{n} \cdot N+b\left(\frac{B_{1}}{c \cdot \phi}-N\right) \cdot \phi
$$

as $\hat{H}_{2}(\gamma)=b_{n} \cdot(1-\phi) \cdot N$. Regardless of $\phi$, all non-elective patients are treated. Differentiating with respect to $\phi$ yields

$$
\frac{\partial H_{1}}{\partial \phi}=b\left(\frac{B_{1}}{c \cdot \phi}-N\right)-b^{\prime}\left(\frac{B_{1}}{c \cdot \phi}-N\right) \cdot \frac{B_{1}}{c \cdot \phi}
$$


At $\phi=1$ and $B_{1}=B^{*}, e_{1}^{h}=\frac{B^{*}}{c}-N=\frac{B^{*}}{c}-n^{*}=e^{*}$, therefore,

$$
\begin{aligned}
\frac{\partial H_{1}}{\partial \phi}\left(1, B^{*}\right) & =b\left(e^{*}\right)-b^{\prime}\left(e^{*}\right) \cdot\left(e^{*}+n^{*}\right) \\
& =b\left(e^{*}\right)-c \cdot\left(e^{*}+n^{*}\right)
\end{aligned}
$$

which is $\geq 0$ or $<0$ depending on the size of $n^{*}$. If $\frac{\partial H_{1}}{\partial \phi}\left(1, B^{*}\right) \geq 0$, then $\hat{\phi}_{1}^{h}=1$, while if $\frac{\partial H_{1}}{\partial \phi}\left(1, B^{*}\right)<0$, then $\hat{\phi}_{1}^{h}<1$ by the concavity of $H_{1}(\phi)$.

(iii) Let $b_{n}>b^{\prime}(0)>(1+\gamma) \cdot c$. In this case,

$$
H_{1}\left(\phi, B_{1}\right)=b_{n} \cdot N+b\left(\frac{B_{1}}{c \cdot \phi}-N\right) \cdot \phi+b(\hat{e}) \cdot(1-\phi)
$$

as $\hat{H}_{2}(\gamma)=b_{n} \cdot(1-\phi) \cdot N+b(\hat{e}) \cdot(1-\phi)$. Regardless of $\phi$, all non-elective patients are treated. Differentiating with respect to $\phi$ yields,

$$
\frac{\partial H_{1}}{\partial \phi}=b\left(\frac{B_{1}}{c \cdot \phi}-N\right)-b^{\prime}\left(\frac{B_{1}}{c \cdot \phi}-N\right) \cdot \frac{B_{1}}{c \cdot \phi}-b(\hat{e}) .
$$

Therefore,

$$
\frac{\partial H_{1}}{\partial \phi}\left(1, B^{*}\right)=b\left(e^{*}\right)-b^{\prime}\left(e^{*}\right) \cdot\left(e^{*}+n^{*}\right)-b(\hat{e})
$$

If $\gamma=0$, then $e^{*}=\hat{e}$ and $\frac{\partial H_{1}}{\partial \phi}\left(1, B^{*}\right)=-b^{\prime}\left(e^{*}\right) \cdot\left(e^{*}+n^{*}\right)<0$ and $\hat{\phi}_{1}^{h}<1$. If $\gamma \geq \frac{b^{\prime}(0)}{c}-1$, then $\hat{e}=0$, and $\frac{\partial H_{1}}{\partial \phi}\left(1, B^{*}\right)=b\left(e^{*}\right)-b^{\prime}\left(e^{*}\right) \cdot\left(e^{*}+n^{*}\right)$ Clearly, if $b\left(e^{*}\right)-b^{\prime}\left(e^{*}\right) \cdot\left(e^{*}+n^{*}\right)<0$, then $\frac{\partial H_{1}}{\partial \phi}\left(1, B^{*}\right)<0$ and $\hat{\phi}_{1}^{h}<1$. However, if $b\left(e^{*}\right)-b^{\prime}\left(e^{*}\right) \cdot\left(e^{*}+n^{*}\right) \geq 0$, then by continuity there is an $\hat{e}_{c}$ such that $b\left(e^{*}\right)-b\left(\hat{e}_{c}\right)-b^{\prime}\left(e^{*}\right) \cdot\left(e^{*}+n^{*}\right)=0$. Let $\gamma_{c}$ be the $\gamma$ that induces $\hat{e}_{c}$. For $\gamma \geq \gamma_{c}, \frac{\partial H_{1}}{\partial \phi}\left(1, B^{*}\right) \geq 0$ and $\hat{\phi}_{1}^{h}=1$, while for $\gamma<\gamma_{c}, \frac{\partial H_{1}}{\partial \phi}\left(1, B^{*}\right)<0$ and $\hat{\phi}_{1}^{h}<1$ by the concavity of $H_{1}(\phi)$.

4. A Period One Comparative Static: Where the choice of $\phi$ is interior, $\hat{\phi}_{1}^{h}$ makes (A-5) and (A-8) above equal to zero. Applying the Implicit Function Theorem yields

$$
\frac{\partial \hat{\phi}_{1}^{h}}{\partial B_{1}}=\frac{b_{e}^{\prime \prime}(\cdot) \frac{B_{1}}{c^{2} \phi^{2}}}{\frac{\partial^{2} H_{1}}{\partial \phi^{2}}}=\frac{\hat{\phi}_{1}^{h}}{B_{1}}>0 \quad \text { and } \quad \frac{\partial \hat{\phi}_{1}^{h}}{\partial \mathrm{B}_{1}} \cdot \frac{\mathrm{B}_{1}}{\hat{\phi}_{1}^{\mathrm{h}}}=1
$$


by the concavity of $b(\cdot)$ and the result in (A-2) above.

5. Proof of Proposition 2: (i) In Proposition 1, conditions are given under which the public hospital chooses $\hat{\phi}_{1}^{h}=1$ when offered $B^{*}$. Given $B^{*}$ and $\hat{\phi}_{1}^{h}=1$ the public hospital chooses to treat all non-elective patients, $n^{*}=N$, and $e^{*}$ elective patients. These are the welfare maximising choices for $n$ and $e$ and so the government is able to achieve the welfare maximum by offering $\hat{B}_{1}=B^{*}$.

(ii) Differentiating the objective function of (16) in the text with respect to $B_{1}$ yields

$$
\begin{aligned}
\frac{d W_{1}}{d B_{1}} & =b_{n} \cdot N \cdot \frac{d \hat{\phi}_{1}^{h}}{d B_{1}}+b^{\prime}(\cdot) \cdot\left(\frac{1}{c \cdot \hat{\phi}_{1}^{h}\left(B_{1}\right)}-\frac{B_{1}}{c \cdot\left(\hat{\phi}_{1}^{h}\left(B_{1}\right)\right)^{2}} \cdot \frac{d \hat{\phi}_{1}^{h}}{d B_{1}}\right) \cdot \hat{\phi}_{1}^{h}\left(B_{1}\right) \\
& +b(\cdot) \cdot \frac{d \hat{\phi}_{1}^{h}}{d B_{1}}-\frac{d \hat{H}_{2}}{d \phi} \cdot \frac{d \hat{\phi}_{1}^{h}}{d B_{1}}-1-(1+\gamma) \cdot \frac{d \hat{B}_{2}}{d B_{1}} .
\end{aligned}
$$

Rearranging, collecting terms, and using (A-5) or (A-8) above gives

$$
\frac{d W_{1}}{d B_{1}}=\frac{\partial H_{1}}{\partial \phi} \cdot \frac{d \hat{\phi}_{1}^{h}}{d B_{1}}+\frac{b^{\prime}(\cdot)}{c}-1-(1+\gamma) \cdot \frac{d \hat{B}_{2}}{d B_{1}} .
$$

In Proposition 1, conditions are given under which the public hospital chooses $\hat{\phi}_{1}^{h}<1$ when offered $B^{*}$. Assuming an interior solution for $\hat{\phi}_{1}^{h}$ implies $\frac{\partial H_{1}}{\partial \phi}=0$. Using this result

$$
\frac{d W_{1}}{d B_{1}}\left(B^{*}\right)=\frac{b^{\prime}(\cdot)}{c}-1-(1+\gamma) \cdot \frac{d \hat{B}_{2}}{d B_{1}}
$$

Now

$$
\frac{d \hat{B}_{2}}{d B_{1}}= \begin{cases}-c \cdot N \cdot \frac{d \hat{\phi}_{1}^{h}}{d B_{1}}<0 ; & b_{n} \geq(1+\gamma) \cdot c \geq b^{\prime}(0) \\ -c \cdot(\hat{e}+N) \cdot \frac{d \hat{\phi}_{1}^{h}}{d B_{1}}<0 ; & b^{\prime}(0)>(1+\gamma) \cdot c\end{cases}
$$

where the sign comes from (A-10) above. In the text, given $B^{*}$ and $\hat{\phi}_{1}^{h}<1$, it was shown that $\hat{e}_{1}^{h}>e^{*}$. Therefore, $\frac{\hat{e}_{1}^{h}}{\hat{\phi}_{1}^{h}}>e^{*}$. By the concavity of $b(e)$, $b^{\prime}\left(\frac{e_{1}^{h}}{\hat{\phi}_{1}^{h}}\right)<b^{\prime}\left(e^{*}\right)=c$. The last equality follows from the definition of $e^{*}$. As a 
result, $\frac{b^{\prime}\left(\frac{\hat{e}_{1}^{h}}{\hat{\phi}_{1}^{h}}\right)}{c}-1<0$. Therefore the sign of $\frac{d W_{1}}{d B_{1}}\left(B^{*}\right)$ is ambiguous. If this sign is positive, then $\hat{B}_{1}>B^{*}$, while if it is negative $\hat{B}_{1}<B^{*}$ as long as $W_{1}\left(B_{1}\right)$ is concave.

It is shown above that $\frac{\partial \hat{\phi}_{1}^{h}}{\partial B_{1}} \cdot \frac{B_{1}}{\hat{\phi}_{1}^{h}}=1$. Therefore, $\hat{\phi}_{1}^{h}=K \cdot B_{1}$, where $K$ is independent of $B_{1}$ but is a function of the other parameters of the model. In particular, $K(c, \gamma)$. Substitution of $\hat{\phi}_{1}^{h}=K(c, \gamma) \cdot B_{1}$ into (A-13) above and rearranging gives

$$
\frac{d W_{1}}{d B_{1}}=b^{\prime}\left(\frac{1}{c \cdot K(c, \gamma)}-N\right) \cdot \frac{1}{c}-1+(1+\gamma) \cdot c \cdot(\hat{e}+N) \cdot K(c, \gamma) .
$$

This is independent of $B_{1}$. Therefore, $W_{1}$ is a linear function of $B_{1}$ and, depending on the parameters of the model, can be increasing or decreasing. If it is decreasing, then the optimal period 1 budget is $\hat{B}_{1}=0$, while if it is increasing, $\hat{B}_{1}$ is chosen so that the public hospital finds it optimal to set $\hat{\phi}_{1}^{h}=1$.

Numerical Example 1: Specifically, for $b_{n}=120, b(e)=100 \cdot e-\frac{1}{2} \cdot e^{2}$, $c=40, E=100, N=30$ and $\gamma=\frac{1}{4}$, it can be shown that $\frac{d W_{1}}{d B_{1}}>0$ all $B_{1}$ including $B^{*}$ so $\hat{B}_{1}=4800>B^{*}=3600$. On the other hand if $\gamma$ is lower, in particular, $\gamma=\frac{1}{20}$, then it can be shown that $\frac{d W_{1}}{d B_{1}}<0$ so $\hat{B}_{1}=0<B^{*}$. Therefore, $\hat{B}_{1}$ can be greater or less than $B^{*}$.

6. Concavity of $\check{H}_{1}(\phi)$. Differentiating (19) of the text with respect to $\phi$ yields

$$
\begin{aligned}
\frac{\partial \check{H}_{1}}{\partial \phi} & =b_{n} \cdot N+b\left(\frac{B_{1}}{c \cdot \phi}-N\right)-b^{\prime}\left(\frac{B_{1}}{c \cdot \phi}-N\right) \cdot \frac{B_{1}}{c \cdot \phi} \\
& +\frac{d \check{H}_{2}(\phi)}{d \phi} .
\end{aligned}
$$

In the interval $\phi_{n} \leq \phi \leq \phi_{e}$

$$
\frac{\partial^{2} \check{H}_{1}}{\partial \phi^{2}}=b_{e}^{\prime \prime}(\cdot) \cdot \frac{B_{1}^{2}}{c^{2} \phi^{3}}<0
$$


by the concavity of $b(\cdot)$, while in the interval $\phi>\phi_{e}$

$$
\begin{aligned}
\frac{\partial^{2} \check{H}_{1}}{\partial \phi^{2}} & =b_{e}^{\prime \prime}(\cdot) \cdot \frac{B_{1}^{2}}{c^{2} \phi^{3}}+\left(-2 b^{\prime}(\check{e})+(1-\phi) \cdot b_{e}^{\prime \prime}(\check{e}) \frac{d \check{e}}{d \phi}\right) \cdot \frac{d \check{e}}{d \phi} \\
& +(1-\phi) \cdot b^{\prime}(\hat{e}) \cdot \frac{d^{2} \check{e}}{d \phi^{2}}<0
\end{aligned}
$$

by the concavity of $b(\cdot)$, as long as $\frac{d^{2} \hat{e}}{d \phi^{2}}$ is not too positive.

7. Proof of Proposition 3: (i) At $\phi=1$ and $B_{1}=B^{*}, \check{e}=e^{*}$ as $\gamma(1)=0$. Therefore, $\frac{\partial \check{H}_{1}}{\partial \phi}\left(1, B^{*}\right)=-b^{\prime}\left(e^{*}\right) \cdot\left(e^{*}+n^{*}\right)<0$ and $\check{\phi}_{1}^{h}<1$.

(ii) Given $B^{*}=c \cdot\left(e^{*}+N\right)>c \cdot \phi_{n} \cdot N$, all $\phi \leq \phi_{n}$ are feasible choices for the public hospital. At $\left(\phi_{n}, B^{*}\right)$,

$$
\check{H}_{1}\left(\phi_{n}, B^{*}\right)=b_{n} \cdot \phi_{n} \cdot N+b\left(\frac{B^{*}}{c \cdot \phi_{n}}-N\right)+b_{n}\left(1-\phi_{n}\right) \cdot N .
$$

This is strictly greater than $\check{H}_{1}\left(\phi^{\prime}, B^{*}\right)$ where $\phi^{\prime}<\phi_{n}$ because $b_{n}>b^{\prime}(0)$ and $\check{H}_{1}\left(\phi_{n}, B^{*}\right)$ has an extra positive term $\left(b_{n} \cdot\left(1-\phi_{n}\right) \cdot N\right)$. Therefore, the public hospital chooses $\phi_{n} \leq \check{\phi}_{1}^{h}$.

(iii) From (19) and (A-16) of the text, at $\phi_{e}$ the left hand side derivative of $\check{H}_{1}\left(\phi, B^{*}\right)$ is given by

$$
\frac{\partial \check{H}_{1}}{\partial \phi}\left(\phi_{e}^{-}, B^{*}\right)=b\left(\frac{B^{*}}{c \cdot \phi_{e}}-N\right)-b^{\prime}\left(\frac{B^{*}}{c \cdot \phi_{e}}-N\right) \cdot \frac{B^{*}}{c \cdot \phi_{e}}
$$

as $\check{H}_{2}(\phi)=b_{n} \cdot(1-\phi) \cdot N$. On the other hand, the right hand side derivative is given by

$$
\frac{\partial \check{H}_{1}}{\partial \phi}\left(\phi_{e}^{+}, B^{*}\right)=b\left(\frac{B^{*}}{c \cdot \phi_{e}}-N\right)-b^{\prime}\left(\frac{B^{*}}{c \cdot \phi_{e}}-N\right) \cdot \frac{B^{*}}{c \cdot \phi_{e}}+\left(1-\phi_{e}\right) \cdot b^{\prime}(0) \cdot \frac{d \check{e}}{d \phi}
$$

as $\check{e}=0$. If $\frac{\partial \check{H}_{1}}{\partial \phi}\left(\phi_{e}^{-}, B^{*}\right)>0$, then $\phi_{e}<\check{\phi}_{1}^{h}$ by the concavity of $\check{H}_{1}(\phi)$ over the interval $\left[\phi_{n}, \phi_{e}\right]$ and the fact that $\left(1-\phi_{e}\right) \cdot b^{\prime}(0) \cdot \frac{d \check{e}}{d \phi}>0$. If $\frac{\partial H_{1}}{\partial \phi}\left(\phi_{e}^{+}, B^{*}\right) \leq 0$, then $\check{\phi}_{1}^{h} \leq \phi_{e}$ by the concavity of $\check{H}_{1}(\phi)$ over the interval $\left(\phi_{e}, 1\right]$ 


\section{Another Comparative Static:}

Applying the Implicit Function Theorem to (A-16) above yields

$$
\frac{d \check{\phi}_{1}^{h}}{d B_{1}}=\frac{b_{e}^{\prime \prime}(\cdot) \frac{B_{1}}{c^{2}\left(\check{\phi}_{1}^{h}\right)^{2}}}{\frac{\partial^{2} \check{H}_{1}}{(\partial \phi)^{2}}}>0
$$

by the concavity of $b(\cdot)$ and the results in 6 above.

\section{Proof of Proposition 4:}

(i) Given $b_{n}>b^{\prime}(0)>c$, if $\check{B}_{1}<c \cdot \phi_{n} \cdot N$, then the public hospital only treats non-elective patients and the government could increase welfare by increasing $B_{1}$ and having more non-elective patients treated.

(ii) Differentiating (20) in the text with respect to $B_{1}$ and applying the envelope theorem yields an expression identical to (A-13) above except there are two additional terms. In particular,

$$
\frac{d \check{W}_{1}}{d B_{1}}=\frac{b^{\prime}(\cdot)}{c}-1-\left(1+\gamma\left(\check{\phi}_{1}^{h}\left(B_{1}\right)\right)\right) \cdot \frac{d \check{B}_{2}}{d B_{1}}-\check{B}_{2}\left(\check{\phi}_{1}^{h}\left(B_{1}\right)\right) \cdot \frac{d \gamma}{d \phi} \cdot \frac{d \check{\phi}_{1}^{h}}{d B_{1}},
$$

where

$$
\frac{d \check{B}_{2}}{d B_{1}}= \begin{cases}-c \cdot N \cdot \frac{d \check{\phi}_{1}^{h}}{d B_{1}}<0 ; & \phi_{n} \leq \phi\left(B_{1}\right) \leq \phi_{e} \\ -c \cdot(N+\check{e}(\phi)) \cdot \frac{d \check{\phi}_{1}^{h}}{d B_{1}}+ & \\ c \cdot\left(1-\check{\phi}_{1}^{h}\left(B_{1}\right)\right) \cdot \frac{d \check{e}}{d \phi} \cdot \frac{d \check{\phi}_{1}^{h}}{d B_{1}} ; & \phi_{e}<\phi\left(B_{1}\right) .\end{cases}
$$

The additional terms are

$$
\left[-\check{B}_{2}\left(\check{\phi}_{1}^{h}\left(B_{1}\right)\right)-\left(1+\gamma\left(\check{\phi}_{1}^{h}\left(B_{1}\right)\right)\right) \cdot c \cdot\left(1-\check{\phi}_{1}^{h}\left(B_{1}\right)\right) \cdot \frac{d \check{e}}{d \gamma}\right] \cdot \frac{d \gamma}{d \phi} \cdot \frac{d \check{\phi}_{1}^{h}}{d B_{1}} .
$$

Now $\frac{d \gamma}{d \phi} \cdot \frac{d \check{\phi}_{1}^{h}}{d B_{1}}<0$ so the sign of $(\mathrm{A}-25)$ is the same as the sign of

$$
\check{B}_{2}\left(\check{\phi}_{1}^{h}\left(B_{1}\right)\right)+\left(1+\gamma\left(\check{\phi}_{1}^{h}\left(B_{1}\right)\right)\right) \cdot c \cdot\left(1-\check{\phi}_{1}^{h}\left(B_{1}\right)\right) \cdot \frac{d \check{e}}{d \gamma}
$$

The first additional term in (A-26) is positive while the second additional term is negative. As in the proof of Proposition $2, \frac{b^{\prime}(\cdot)}{c}-1<0$ and 
$\left(1+\gamma\left(\check{\phi}_{1}^{h}\left(B_{1}\right)\right)\right) \cdot c \cdot(N+\check{e}(\phi)) \cdot \frac{d \check{\phi}_{1}^{h}}{d B_{1}}>$ so the sign of $\frac{d \check{W}_{1}}{d B_{1}}\left(B^{*}\right)$ is still ambiguous.

If $B_{1}$ is such that the public hospital chooses $\phi$ to be in the region $\phi_{n} \leq \phi \leq \phi_{e}$, then (A-17) and (A-22) together imply that $\frac{\partial \check{\phi}_{1}^{h}}{\partial B_{1}} \cdot \frac{B_{1}}{\tilde{\phi}_{1}^{h}}=1$. Therefore, $\check{\phi}_{1}^{h}=K(\cdot) B_{1}$. Substituting this into (A-23) yields

$$
\begin{aligned}
\frac{d \check{W}_{1}}{d B_{1}} & =b^{\prime}\left(\frac{1}{c \cdot K(\cdot)}-N\right) \cdot \frac{1}{c}-1+\left(1+\gamma\left(K(\cdot) B_{1}\right)\right) \cdot c \cdot N \cdot K(\cdot) \\
& -\frac{d \gamma}{d \phi}\left(K(\cdot) B_{1}\right) \cdot K(\cdot) \cdot c \cdot\left(1-K(\cdot) B_{1}\right) \cdot N .
\end{aligned}
$$

Unlike in sub-section 3.2, this derivative is a function of $B_{1}$ and contains an extra positive term. This extra term can make $\frac{d \breve{W}_{1}}{d B_{1}}>0$ for all $B_{1}$ that yield $\phi$ in the specified region. In this case, the optimal period 1 budget is such that $\phi_{e} \leq \check{\phi}_{1}^{h}\left(\check{B}_{1}\right)$.

Numerical Example 2: The functions and parameters are as given in numerical example 1 except $\gamma=\gamma_{0}-\gamma_{0} \cdot \phi$.

Let $\gamma_{0}=3$ : In this case $\phi_{n}=\frac{1}{3}$ and $\phi_{e}=\frac{1}{2}$. Calculation reveals that $\frac{d \check{W}_{1}}{d B_{1}}\left(B^{*}\right)>0, \check{B}_{1}=3776>B^{*}=3600$, and $\check{\phi}_{1}^{h}\left(\check{B}_{1}\right)=\frac{86}{100}>\phi_{e}$.

Let $\gamma_{0}=10$ : In this case $\phi_{n}=\frac{8}{10}$ and $\phi_{e}=\frac{17}{20}$. Calculation reveals that $\frac{d \check{W}_{1}}{d B_{1}}\left(B^{*}\right)<0, \check{B}_{1}=3589<B^{*}=3600$, and $\check{\phi}_{1}^{h}\left(\check{B}_{1}\right)=\frac{94}{100}>\phi_{e}$.

10. Proof of Proposition 5: From (17) in the text, at $\check{e}_{2}, b^{\prime}\left(\frac{\check{e}_{2}}{1-\phi}\right)-(1+$ $\gamma(\phi)) \cdot c=0$. Therefore, the first two terms in the first row of (25) are together negative because $e_{2}^{m}\left(\check{e}_{2}\right)$ is positive and $b(\cdot)$ is concave. In addition, the third term in the first row of (25) is also negative because the term in brackets is positive (the monopoly price is greater than marginal cost) while $e_{2}^{m^{\prime}}\left(e_{2}\right)$ is negative. Therefore, at $\check{e}_{2}, \frac{\partial \tilde{W}_{2}}{\partial e_{2}}<0$. Given $\tilde{W}_{2}$ is concave, then $\tilde{e}_{2}<\check{e}_{2}$. 
If $\tilde{e}_{2}+e_{2}^{m}\left(\tilde{e}_{2}\right)=\check{e}_{2}$, then the first two terms in the first row of (25) are together zero. The third term is negative, so $\frac{\partial \tilde{W}_{2}}{\partial e_{2}}<0$. As a result, $\tilde{e}_{2}+e_{2}^{m}\left(\tilde{e}_{2}\right)<\check{e}_{2}$.

11. Proof of Proposition 6: Comparing (A-16) and (26) in the text and using the results that $\tilde{e}<\check{e}, b(\tilde{e})<b(\check{e})$, and $b^{\prime}(\tilde{e})>b^{\prime}(\check{e})$ establishes that a sufficient condition for the derivative in (26) to be greater than zero at $\check{\phi}_{1}^{h}$ is $\frac{d \tilde{e}}{d \phi}=\frac{d \check{e}}{d \gamma} \cdot \frac{d \gamma}{d \phi}>\frac{d \check{e}}{d \phi}=\frac{d \tilde{e}}{d \gamma} \cdot \frac{d \gamma}{d \phi}$. Now

$$
\frac{d \check{e}}{d \gamma}=\frac{c}{b^{\prime \prime}(\check{e})}
$$

while

$$
\frac{d \tilde{e}}{d \gamma}=\frac{c}{b^{\prime \prime}\left(\tilde{e}+e^{m}(\tilde{e})\right) \cdot\left(1+\frac{d e^{m}}{d e}\right)^{2}}
$$

If $b^{\prime}(e)$ is a linear function, that is $b^{\prime \prime \prime}(e)=0$, then $b^{\prime \prime}(\check{e})=b^{\prime \prime}\left(\tilde{e}+e^{m}(\tilde{e})\right)$. As $-1<\frac{d e^{m}}{d e}<0, \frac{d \tilde{e}}{d \gamma}>\frac{d \check{e}}{d \gamma}$. Therefore, at $\check{\phi}_{1}^{h}, \frac{\partial \tilde{H}_{1}}{\partial \phi}>0$ and $\tilde{\phi}_{1}^{h}>\check{\phi}_{1}^{h}$.

12. Sign of $\frac{d \tilde{W}_{1}}{d B_{1}}\left(\check{B}_{1}\right)$.

Differentiating $(27)$ of the text and evaluating at $\check{B}_{1}$ yields

$$
\begin{aligned}
\frac{d \tilde{W}_{1}}{d B_{1}}\left(\check{B}_{1}\right) & =b^{\prime}\left(\frac{\check{B}_{1}}{c \cdot \tilde{\phi}_{1}^{h}\left(\check{B}_{1}\right)}-N\right) \cdot \frac{1}{c}-1-\left(1-\gamma\left(\tilde{\phi}_{1}^{h}(\cdot)\right)\right) \cdot \frac{d \tilde{B}_{2}}{d B_{1}}-\tilde{B}_{2}\left(\tilde{\phi}_{1}^{h}(\cdot)\right) \frac{d \gamma}{d B_{1}} \\
& +\left[b\left(\frac{\check{B}_{1}}{c \cdot \tilde{\phi}_{1}^{h}\left(\check{B}_{1}\right)}-N\right)-b^{\prime}\left(\frac{\check{B}_{1}}{c \cdot \tilde{\phi}_{1}^{h}\left(\check{B}_{1}\right)}-N\right) \cdot \frac{\check{B}_{1}}{c \cdot \tilde{\phi}_{1}^{h}\left(\check{B}_{1}\right)}+c \cdot e^{m}(\tilde{e})\right. \\
& \left.-b\left(\tilde{e}\left(\tilde{\phi}_{1}^{h}\right)+e^{m}(\tilde{e})\right)+\left(1-\tilde{\phi}_{1}^{h}\left(\check{B}_{1}\right)\right) \cdot b^{\prime}\left(\tilde{e}\left(\tilde{\phi}_{1}^{h}\right)+e^{m}(\tilde{e})\right) \cdot \frac{d \tilde{e}}{d \phi}\right] \cdot \frac{d \tilde{\phi}_{1}^{h}}{d B_{1}} \\
& +\left(1-\phi\left(B_{1}\right)\right) \cdot\left(b^{\prime}\left(\tilde{e}\left(\tilde{\phi}_{1}^{h}\right)+e^{m}(\tilde{e})\right)-c\right) \cdot \frac{d e^{m}}{d \phi} \cdot \frac{d \tilde{\phi}_{1}^{h}}{d B_{1}}
\end{aligned}
$$

Unlike in section 3 , the term in square brackets does not equal zero. In fact, setting (26) in the text equal to zero implies the term in square brackets is positive because $\left(\tilde{e}\left(\tilde{\phi}_{1}^{h}\right)+e^{m}(\tilde{e})\right)<\check{e} .(\mathrm{A}-30)$ is a very complicated expression and its sign at $\check{B}_{1}$ is ambiguous. 
Numerical Example 3: The functions and parameters are as given in numerical example 2. Note that $B^{*}=3600, e^{*}=60, n^{*}=N=30$, and $W^{*}=4200$.

Let $\gamma_{0}=3$ :

$\tilde{B}_{1}=3323<B^{*}<\check{B}_{1}=3776$,

$\tilde{\phi}_{1}^{h}=.89>\check{\phi}_{1}^{h}=.86$,

$\tilde{W}_{1}=4098>\check{W}_{1}=3862$,

$\tilde{\phi}_{1}^{h} \cdot e^{*}=53.4<\tilde{e}_{1}=56.5<\check{e}_{1}=68.5$,

$\tilde{e}_{2}+\tilde{e}_{2}^{m}=.66+3=3.66<\check{e}_{2}=5.92$.

Let $\gamma_{0}=10$ :

$\tilde{B}_{1}=3508<B^{*}<\check{B}_{1}=3598$,

$\tilde{\phi}_{1}^{h}=.96>\check{\phi}_{1}^{h}=.94$,

$\tilde{W}_{1}=4168>\check{W}_{1}=4056$,

$\tilde{\phi}_{1}^{h} \cdot e^{*}=57.6<\tilde{e}_{1}=58.8<\check{e}_{1}=61.7$,

$\tilde{e}_{2}+\tilde{e}_{2}^{m}=.1+1=1.1<\check{e}_{2}=2.2$. 